\title{
A method for effect modifier assessment (EMA) in ergonomic intervention research
}

\section{Edwards, Kasper; Winkel, Jørgen}

\section{Published in:}

Applied Ergonomics

Link to article, DOI:

10.1016/j.apergo.2018.05.007

Publication date:

2018

Document Version

Peer reviewed version

Link back to DTU Orbit

\section{Citation (APA):}

Edwards, K., \& Winkel, J. (2018). A method for effect modifier assessment (EMA) in ergonomic intervention research. Applied Ergonomics, 72, 113-120. https://doi.org/10.1016/j.apergo.2018.05.007

\section{General rights}

Copyright and moral rights for the publications made accessible in the public portal are retained by the authors and/or other copyright owners and it is a condition of accessing publications that users recognise and abide by the legal requirements associated with these rights.

- Users may download and print one copy of any publication from the public portal for the purpose of private study or research.

- You may not further distribute the material or use it for any profit-making activity or commercial gain

- You may freely distribute the URL identifying the publication in the public portal

If you believe that this document breaches copyright please contact us providing details, and we will remove access to the work immediately and investigate your claim 


\title{
A method for effect modifier assessment (EMA) in ergonomic intervention research
}

\author{
Kasper Edwards ${ }^{1 *}$ and Jörgen Winkel ${ }^{1,2}$ \\ ${ }^{1}$ DTU Management Engineering \\ Building 424 \\ Technical University of Denmark \\ DK-2800 Lyngby, Denmark \\ Email: kaed@dtu.dk \\ ORCID: 0000-0002-6328-1765 \\ ${ }^{*}$ Corresponding author ${ }^{2}$ \\ Department of Sociology and Work Science \\ University of Gothenburg \\ Box 705, SE-405 30 Gothenburg, Sweden \\ Email: jorgen.winkel@gu.se \\ ORCID: 0000-0001-7993-396X
}




\section{Abstract}

The numerous opportunities for effect modifications pose a major challenge in ergonomic intervention research. Even studies in systematic reviews that are assessed as being of high quality generally lack any proper consideration of the potential effect modifiers. We have developed a method for effect modifier assessment (EMA) in intervention research. The EMA method uses a participatory workshop consisting of representatives from all occupational groups in the investigated organization. The workshop identifies both intervention and modifier events. These are categorized into themes, then analyzed and evaluated for their potential effects on the investigated outcomes. The overall impact of the pooled modifier themes is finally estimated in relation to the estimated impact of the intervention events. In the present study, the EMA method was tested in two cases. The findings suggest that it provides information that strengthens inferences about the impact of the investigated ergonomic interventions. Further evaluation of the method is recommended. 
Highlights (three items to be placed in a separate file)

- The method identifies potential effect modifiers in ergonomic intervention research.

- The method estimates the potential impact of both interventions and modifiers.

- The method is tested in two cases; the potential effect modification is identified.

Keywords: Ergonomic intervention, effect modification, research method 


\section{Introduction}

Risk factors for impaired musculoskeletal and mental health still seem to be the main work environment issues in working life (Parent-Thirion et al, 2017). Ergonomic intervention research aimed to reduce these problems includes studies in which researchers follow or arrange changes in working conditions to determine the effects on risk factors and/or health. To demonstrate causality, such case studies should meet numerous scientific quality criteria (see, e.g., Kristensen, 2005; Westgaard and Winkel, 1997). Several systematic reviews of ergonomic intervention research have adopted the "best evidence synthesis" approach (Slavin, 1995). The ambitious systematic review by Kennedy et al. (2010) used this procedure to assess the role of occupational health and safety interventions in the prevention of upper extremity disorders. They identified more than 15,000 articles, 14 of which were classified as "high-quality studies" based on many scientific quality criteria. All 14 were randomized controlled trials (RCTs) which, inter alia, means that a control group was included.

Research assessing the impact of ergonomic interventions may be challenged by factors other than those considered by Kennedy et al. (2010). The systematic review by Westgaard and Winkel (2011) showed that changes in and around a production system, e.g. restructuring, downsizing, management style, and parallel interventions, may impact musculoskeletal and mental health and/or the corresponding risk factors. Such changes may affect the investigated outcome in the case/intervention groups and may therefore become effect modifiers. Furthermore, any differences in effect modification between case and control groups may also hide potential intervention effects. Effect modification was not investigated in any of the 14 case studies classified as "high quality" by Kennedy et al. (2010). The same goes for many other "high-quality" ergonomic intervention studies (see, e.g., the systematic ergonomic reviews by Driessen et al., 2010 and Leyshon et al., 2010).

This narrow view of what constitutes "high quality" has been questioned by Westgaard (2010), who calls for other methods than RCTs to evaluate ergonomic interventions that also consider effect modifiers. The "process evaluation tool" presented by Nielsen et al. (2006) and the "critical incident technique" (Flanagan, 1954) consider contextual factors and might therefore be able to identify potential effect modifiers. However, we have not been able to identify any intervention studies using these tools for the assessment of potential effect modifiers. Neither did our literature review identify any other methods for this purpose.

To address this issue, we developed a new method for $\underline{\boldsymbol{e}}$ ffect $\underline{\text { modifier }} \underline{\boldsymbol{a}}$ ssessment (the EMA method) in ergonomic intervention studies. In the EMA method, a workshop is held to estimate both modifier and intervention events and their impact on the investigated outcome(s). This paper presents the method and includes a preliminary examination of its applicability in two intervention case studies at a Danish hospital.

\section{The EMA method}

The proposed EMA method consists of a EMA workshop and subsequent analyses. Facilitated by a coach, the workshop is used to collect information from employees about their perceptions of past 
significant events in their workplace that may have acted as modifiers of their perceived change of the work environment during the time period of an intervention. The data were analyzed to obtain semi-quantitative estimations of the impact of the events on the intervention outcome. Our proposed method was inspired by the chronicle workshop methodology, presented by Limborg and Hvenegaard (2011), and the focus group depth interview method (Goldman, 1962; McLafferty 2004; Millward, 2012). The EMA method was developed to assess potential modifiers of physical and psychosocial aspects of the work environment (WE) (cf. Westgaard and Winkel 2011).

\subsection{Description of the method}

\subsubsection{General procedure}

During the EMA workshop, the participants identify potential modifiers (events) that they perceive as having an impact on their work or work situation. Whether the events are individually or collectively experienced is also determined. Event identification recall is enhanced by creating a timeline for the period during which the intervention study is performed.

During the workshop, participants answer three questions about the important events/changes during the intervention project time period. The first question focuses on important changes/events:

Question 1: Think back in time. What important changes/events related to your workplace/work have occurred during the intervention project time period?

The second question focuses on events related to changes to work processes and equipment:

Question 2: Think back in time. What important changes/events related to work processes and equipment at your workplace/work have occurred during the intervention project time period?

The third question focuses on changes to the work environment;

Question 3: Think back in time. What important changes/events related to the work environment have occurred during the intervention project time period (outcome)?

The participants write their answers on post-it-notes, including only one event on each post-it note. The answers are then discussed collectively, and the employees' perceived impact on the work environment is assessed.

\subsubsection{Workshop participants}

Depending on size of the investigated organization and the number of occupational groups involved, three to five people participate in EMA workshops. It is important to include representatives from all occupational groups involved in the investigated intervention, and they should have appropriate experience working in the organization. According to McLafferty (2004), the selected participants should know one another, as this may facilitate the group process. In large organizations, it may be necessary to conduct several EMA workshops to identify all changes/events. 


\subsubsection{Practical procedure}

For the workshop, a room with seating and writing facilities is needed allowing the participants to work undisturbed. The workshop facilitator (coach/researcher) draws a project timeline on a wall or whiteboard. Post-it-notes are attached to the timeline indicating when the assessed events occurred (see Figure 1). The workshop should be audio recorded. The participants' responses are later transcribed and analyzed.

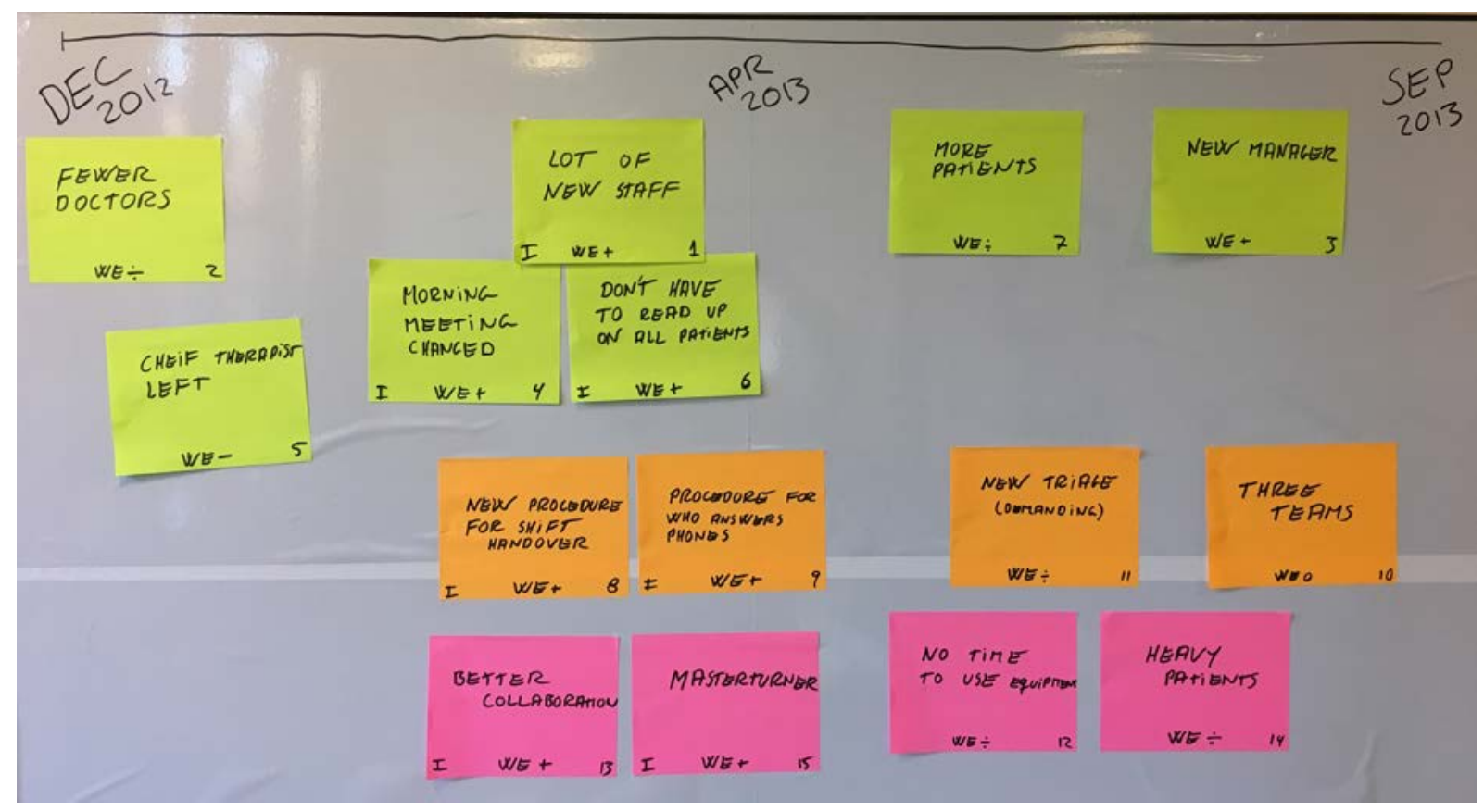

Figure 1. An illustration of events placed on a timeline during an EMA workshop. Interpretation of each event requires dialogue with and between participants (cf. section 2.d.iv and 2.d.v).

The different colors represent the three questions answered during the workshop; yellow/top: Question 1; orange/middle: Question 2; purple/bottom: Question 3, see section 2.1.1. Post-it notes marked with "I" indicate events that are part of the intervention. WE: work environment +:better, 0:same, -:worse.

The EMA workshop consists of the following activities:

1. Instructions presented by the coach to the workshop participants

a. The purpose of the workshop is to map significant events that have occurred at or around the department during the investigated time period. The focus on the WE should not be mentioned until Question 3 is presented (see Section 2.1.1).

b. The workshop is audio-recorded. The participants' responses are later transcribed and analyzed. All information is handled anonymously.

c. An "important event" is defined as a change or event that implies an impact on the participants' work situation.

d. Only one event should be written on each post-it-note.

e. The answers on the post-it-notes should be written individually; participants should not discuss their responses with their peers in the workshop.

f. Sufficient time will be given for participants to write down all the important events. This usually lasts $5-10$ minutes.

\section{Workshop actions}


a. The coach distributes the first question and reads it out loud.

b. The participants write their events on the post-it-notes.

c. The coach collects all the post-it-note events from the participants when they have finished writing them.

d. The coach processes one post-it-note at a time by doing the following:

i. Reading the event out loud to the participants

ii. Writing an event number on the post-it-note and reading the event number out loud (for the audio recording and later analysis of the transcription)

iii. Asking the participant when the event initially occurred during the project timeline, and attaching that information to the post-it-note, accordingly

iv. Asking the participant to explain how he/she experienced the event and how the event affected the work situation individually and/or his/her colleagues

v. Asking the other participants to contribute how they experienced the event and how the event affected their work situation and/or their colleagues

vi. Asking the other participants if they have post-it-notes pertaining to the same event. If so, the coach collects the post-it-notes and places them in a pool on the timeline (pooled events). When no more comments are offered, the coach proceeds to the next post-it-note and repeats the process from step 2.d.i. The coach uses different-colored post-it-notes for each of the three questions, and repeats steps 2.d.i-vi.

e. When all three questions have been processed, the coach proceeds to step 3 .

3. Evaluation of the processed responses in cooperation with the workshop coach

a. The coach asks the participants to assess which events have been part of the intervention and to mark the corresponding post-it-note with an "I" for "Intervention."

b. The coach then asks the participants to assess all the events with regard to the perceived impact on the physical WE (PhWE +,0,-) and the psychosocial WE (PSWE $+, 0,-)$, and finally writes the assessments on the corresponding post-it-notes.

\section{Collection of the data}

a. The coach photographs the timeline, including all post-it-notes, for later analysis. Figure 1 illustrates an example.

b. The coach collects all the post-it-notes for later validation, keeping the pooled events together. 


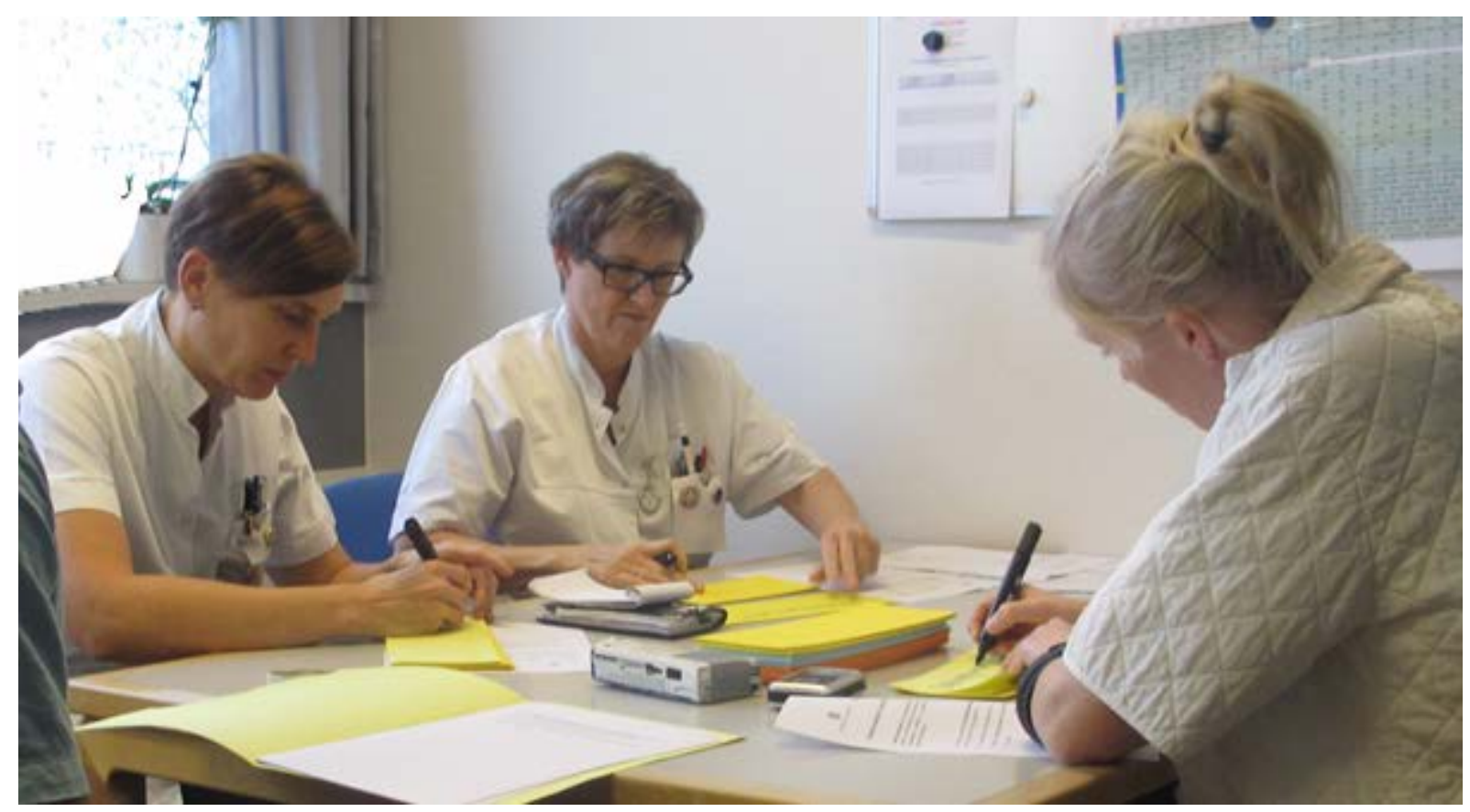

Figure 2. Healthcare professionals writing on post-it notes during an EMA workshop.

\subsubsection{Post-workshop data organization and processing}

All the events are entered into a database for coding and statistical analyses, as indicated in Table 1 below. We recommend using the following set of data fields (Table 1).

Table 1 List of Data Fields in a Database for Statistical Analysis of the Data

\begin{tabular}{|c|l|l|l|}
\hline Data field & Label & Data & Description \\
\hline 1 & Number & Number & $\begin{array}{l}\text { Unique identification number for each event in the database } \\
\text { numbered consecutively stating at 1 }\end{array}$ \\
\hline 2 & Organization & Name & Descriptive name of the organization \\
\hline 3 & Event number & Number & Number written on the post-it-note at workshop step 2.d.ii \\
\hline 4 & Description & Sentence & Description written on the post-it-note at workshop step 2.b \\
\hline 5 & Intervention & Yes/no & $\begin{array}{l}\text { Denotes whether the event was part of the investigated } \\
\text { intervention, assessed during the workshop at step 3.a }\end{array}$ \\
\hline 6 & Psychosocial WE & $-, 0,+$, NA & Impact on the psychosocial WE, assessed at workshop step 3.b \\
\hline 7 & Physical WE & $-, 0,+, N A$ & Impact on the physical WE, assessed at workshop step 3.b \\
\hline 8 & Inclusion & Yes/no & $\begin{array}{l}\text { Events that do not match the definition explained at workshop } \\
\text { step 1.c or NA are excluded from the analysis. }\end{array}$ \\
\hline 9 & Impact on WE & $-, 0,+, N A$ & Combined impact of the psychosocial WE and the physical WE \\
\hline 10 & Theme & Sentence & Descriptive name for the pooled events \\
\hline
\end{tabular}

WE: Work Environment; NA: Not Assessable

Post-workshop data preparation is performed in five steps, as follows:

\section{Transcription of the audio recording}

Data fields 1-7 are completed by entering information on all the collected post-it-notes into the 
database. This is done in the same order and numbering as occurred in the workshop to facilitate the identification of relevant parts of the audio recording.

\section{Exclusion of invalid data}

Data field 8, "Inclusion," is evaluated by estimating whether the event matches the inclusion criteria (i.e., as a change or event that implies an impact on the participants' work situation). Participants sometimes write what they wish had happened and not what actually occurred; those events are not valid and are excluded from further analysis. The included events are marked with, "Yes," and the excluded events are marked with "No." Not assessable (NA) events are excluded.

\section{Reassessment of the WE}

Data field 5, "Intervention," field 6, "Psychosocial WE," and field 7, "Physical WE," are reassessed using triangulation (Jick, 1979). This is based on scientific evidence, researchers' knowledge, the transcribed audio recording from the workshop, the project plan, the action plan, and other available sources. The assessments should be performed by at least two researchers. If an implementation is not yet completed according to the action plan, the assessment is based on the expected effect referring to available evidence from the literature.

\section{Determination of the impact on the WE}

Data field 9, "Impact on the WE," is the combined assessment of field 6, "Psychosocial WE," and field 7, "Physical WE." The WE is assessed as positive (+) for the following combinations of impact on PhWE and PsWE: PhWE+/PsWE +, PhWEO/PsWE+, and PhWE+/PsWEO. The WE impact is assessed as negative (-) for PhWE-/PsWE-, PhWEO/PsWE-, and PhWE-/PsWE0. The WE impact is assessed as zero for PhWEO/PsWEO, PhWE+/PsWE -, and PhWE-/PsWE +.

\section{Development of themes}

Events are pooled into larger themes if they are part of a larger main event or if they refer to the same basic event (Table 1, data field 10). All events within a theme need to have an identical direction of impact on the WE (Table 1, data field 9) and belong to the same class of events (i.e., "Intervention" or "Modifier"). Each theme is given a descriptive name and added to the database, field 10 "Theme". Practical examples are provided in Section 4 below.

\subsubsection{Data analysis}

We suggest the following analyses to provide a descriptive overview and assessment of the extent to which modifiers impact the organization in relation to the assessed impact of the interventions.

\subsubsection{Descriptive statistics}

The data are presented in a table that displays the total number of events, $\mathrm{N}$, retrieved from a workshop (Table 2). The events, and their impact on the WE, are divided into events that are due to the investigated intervention and those that are not (i.e., the effect modifiers).

Table 2 Suggested table for reporting the Number of Events in a Single Case Study due to Interventions and Modifiers and According to WE Impact

\begin{tabular}{|c|l|l|l|l|}
\hline $\mathrm{N}=$ total \# events & & & & \\
\hline Class of event & Events & WE- & WE0 & WE+ \\
\hline Intervention & & & & \\
\hline Modifier & & & & \\
\hline
\end{tabular}




\subsubsection{Assessing the impact of the themes}

Each theme is assessed according to: 1) the amount of staff impacted (minor/medium/major), 2) the amount of work time for the impacted employees (\#1 above) (minor/medium/major), and 3) the level of impact for the affected employees (\#2 above) (minor/medium/major). Thus, the total WE impact of a theme on an affected individual depends on both (2) and (3). These assessments are determined using the triangulation method (Jinck, 1979) referring to scientific evidence, researchers' knowledge, the transcribed audio recording from the workshop, the project plan, the action plan, and other available sources. This analysis should be performed by two researchers. The results of the assessments are entered into a table (see Table 3). Positive and negative WE impacts are indicated by + and -, respectively, and rated in terms of Minor (+ or -), Medium (++ or --) or Major (+++ or ---). The WEO events are excluded from this table and not used in the further analyses. WE impact is assessed for both modifier and intervention themes.

Table 3 Assessment of Each Theme Regarding the WE Impact

\begin{tabular}{|c|c|c|c|c|c|c|}
\hline $\begin{array}{c}\text { Class of } \\
\text { event }\end{array}$ & WE & Theme & \# Events & $\begin{array}{c}\text { Number } \\
\text { of staff }\end{array}$ & $\begin{array}{c}\text { Amount } \\
\text { of work }\end{array}$ & $\begin{array}{c}\text { Level } \\
\text { of } \\
\text { Impact }\end{array}$ \\
\hline $\begin{array}{c}\text { Intervention/ } \\
\text { Modifier }\end{array}$ & $-/ 0 /+/$ NA & $\begin{array}{c}\text { Descriptive name } \\
\text { e.g. "cutbacks" }\end{array}$ & Number & $+/-$ & $+/-$ & $+/-$ \\
\hline
\end{tabular}

Based on the information in Table 3, the potential modifying effect on investigated intervention(s) is assessed, as illustrated in the following two cases.

\section{Materials and Methods for the EMA Method Field Test}

Two cases (A and B) from hospital wards at a Danish university hospital used the lean tool value stream mapping (VSM) method (Keyte and Locher, 2004; Rother and Shook, 2009) to improve patient flow. At this hospital, the VSM method has been further developed to focus on WE (Winkel et al., 2015).

The EMA workshops (one per case) were held one month after the wards finished their interventions. None of the participants in the EMA workshops had participated in the VSM workshops or had managerial responsibilities. A researcher participated in the EMA workshops, which were audio- and video recorded.

Case A was a neurological department with two separate bed wards, training, and rehabilitation with a total staff of 83 employees. Three participated in the EMA workshop - a nurse, a nurse assistant and a therapist. The purpose of the intervention was to develop a standardized care structure for stabilized patients while improving the WE. A nurse, an assistant nurse, and a therapist participated in the EMA workshop. Doctors only accounted for a marginal part the investigated work flow; therefore, they were excluded from this trial.

Case B was an orthopedic bed ward with a total staff of 32 employees. Three employees participated in the EMA workshop - two nurses and a nurse assistant. The purpose of the interventions was to improve the quality of care during the evening shift, reduce overtime, and improve the documentation procedures and transitions between shifts while improving the WE. A nurse and an assistant nurse participated in the EMA workshop; no other occupational groups were part of the intervention. 
Data were collected from spring 2012 to summer 2014 and thereafter analyzed using Microsoft Excel 2016.

\section{Results}

\subsection{Case A: DK_ERG02}

The EMA workshop lasted two hours and identified 46 events, 30 of which were modifiers (Table 4).

Table 4 Overview of Events in Case A and Their Assessed WE Impact

\begin{tabular}{|c|c|c|c|c|}
\hline $\mathbf{N = 4 6}$ & & & & \\
\hline $\begin{array}{c}\text { Class of } \\
\text { event }\end{array}$ & Events & WE- & WE0 & WE+ \\
\hline Intervention & 16 & 9 & 0 & 7 \\
\hline Modifier & 30 & 25 & 4 & 1 \\
\hline
\end{tabular}

The events were sorted into eight themes (Table 5); three themes were assessed as intervention themes and five as modifier themes. A "new care concept" was the main focus of the Intervention. The events could be divided into two themes having an opposite WE impact: "organizational changes integrating care and therapy" and the "rationalization of meetings and breaks." The first impact consisted of seven events; examples of this include: "closer collaboration between nursing care and therapists," "new meeting structure-patients are included in the interdisciplinary ward round," "good team meeting at 1 o'clock," "interdisciplinary ward round- it's good to have relatives more in focus and included earlier," and "better possibility for nursing care to show/argue for rehabilitating nursing care." The theme "New care concept" affects both nurses and therapists (the majority of staff), but only in situations in which the professional groups work together on a patient, which is a minor part of their working time. The impact level of the theme was assessed as major due to the perceived strong improvement in employee collaboration. The second theme, "the rationalization of meetings and breaks," also related to the new care concept, but covered other aspects and consisted of four events. This theme included events, such as "miss the old treatment planning meeting with patients" and "less time for breaks." These were assessed as having a negative WE impact on all staff, and they affected a major part of their work. This was a substantial change in organizing meetings and breaks; thus, it had a major impact.

The main modifier theme, "Cutbacks," included 14 events. This theme covered events, such as "fewer resources for courses/education," "from 4 to 3 people in each patient team-cutback," "layoffs in the beginning of 2013," and "the clinical supervisor function was cut by one nurse." The theme affected all staff and impacted most areas of their work. The level of impact was assessed as major due to an increased workload and reduced resources.

Table 5 Themes Identified in Case A and Their Assessed WE Impact. Positive and Negative WE impact Are indicated by + and -, Respectively, and Rated in Terms of Minor (+ or -), Medium (++ or --) or Major (+++ or ---).

\begin{tabular}{|c|l|l|c|c|c|c|}
\hline $\begin{array}{c}\text { Class of } \\
\text { event }\end{array}$ & WE & Theme & $\begin{array}{c}\text { Number of } \\
\text { events }\end{array}$ & $\begin{array}{c}\text { Number } \\
\text { of staff }\end{array}$ & $\begin{array}{c}\text { Amount } \\
\text { of work }\end{array}$ & $\begin{array}{c}\text { Level of } \\
\text { Impact }\end{array}$ \\
\hline
\end{tabular}




\begin{tabular}{|c|c|l|c|c|c|c|}
\hline Intervention & + & $\begin{array}{l}\text { New care concept: organizational } \\
\text { changes integrating care and therapy }\end{array}$ & 7 & +++ & + & +++ \\
\hline Intervention & - & $\begin{array}{l}\text { New care concept: rationalization of } \\
\text { meetings and breaks }\end{array}$ & 4 & --- & --- & --- \\
\hline Intervention & - & New management style & 5 & --- & -- & -- \\
\hline & & & 1 & + & + & + \\
\hline Modifier & + & New chief therapist & 14 & --- & --- & --- \\
\hline Modifier & - & Cutbacks & 6 & --- & --- & --- \\
\hline Modifier & - & Personal turnover & 3 & --- & --- & --- \\
\hline Modifier & - & Merger with another department & 2 & --- & --- & --- \\
\hline Modifier & - & Work intensification & & & \\
\hline
\end{tabular}

\subsection{Case B: DK_VSM1}

The EMA workshop lasted 45 minutes; it identified 27 events, 14 of which were modifiers. All but one of the events were assessed to have positive impact on WE (see Table 6).

Table 6 Overview of the Events in Case B According to the WE Impact

\begin{tabular}{|c|c|c|c|c|}
\hline $\mathbf{N}=\mathbf{2 7}$ & & & & \\
\hline Class of event & Events & WE- & WEO & WE+ \\
\hline Intervention & 13 & 0 & 0 & 13 \\
\hline Modifier & 14 & 1 & 0 & 13 \\
\hline
\end{tabular}

The events were sorted into seven themes (Table 7); three were assessed as Intervention themes and four were assessed as Modifier themes. The Intervention theme, "improvement of collaboration in patient care," included the following events: "more help across the two groups-we have become better at helping each other," "a bit better at asking if anyone needs help," and "we have been better at asking for help." This theme affected a majority of the staff, but it only impacted a minor part of their work. When it occurred, it was assessed as having a medium-level, positive impact.

One example of a Modifier theme was "new equipment and training." This included seven events, such as "using the functions of the bed" (the staff received training in using the electronic beds), "mobilizing patients-we have become better at this since we started focusing on this," "back-up-a new and good piece of equipment for mobilizing patients," and "mattresses-getting them packed and sent to cleaning. It was hideous before and now we have a trolley designed especially for the purpose." The theme affected all staff and major parts of their work; thus, the impact level was assessed as major.

Table 7 Themes Identified in Case B and Assessed for Their WE Impact. Positive and Negative WE Impacts Are Indicated by + and -, Respectively, and Rated in Terms of Minor (+ or -), Medium (++ or --) or Major (+++ or ---).

\begin{tabular}{|c|c|l|c|c|c|c|}
\hline $\begin{array}{c}\text { Class of } \\
\text { event }\end{array}$ & WE & Theme & $\begin{array}{c}\text { Number of } \\
\text { events }\end{array}$ & $\begin{array}{c}\text { Number } \\
\text { of staff }\end{array}$ & $\begin{array}{c}\text { Amount } \\
\text { of work }\end{array}$ & $\begin{array}{c}\text { Level of } \\
\text { Impact }\end{array}$ \\
\hline Intervention & + & New procedure for shift handover & 5 & +++ & + & +++ \\
\hline Intervention & + & Structured and coordinated care & 5 & +++ & + & ++ \\
\hline
\end{tabular}




\begin{tabular}{|c|c|l|c|c|c|c|}
\hline Intervention & + & $\begin{array}{l}\text { Improvement of collaboration in } \\
\text { patient care }\end{array}$ & 3 & +++ & + & ++ \\
\hline & & & & & & \\
\hline Modifier & + & New equipment and training & 7 & +++ & +++ & +++ \\
\hline Modifier & + & $\begin{array}{l}\text { More precise task allocation between } \\
\text { employees }\end{array}$ & 5 & +++ & ++ & ++ \\
\hline Modifier & + & New dinner procedure & 1 & +++ & + & + \\
\hline Modifier & - & $\begin{array}{l}\text { More precise task allocation between } \\
\text { employees }\end{array}$ & 1 & - & - & - \\
\hline
\end{tabular}

\section{Discussion}

This paper presents a method (EMA) to identify effect modifiers in ergonomic intervention research with a particular focus on changes in and around a workplace that may affect musculoskeletal and/or mental health and/or the corresponding risk factors during the investigated period.

\subsection{Discussion of the results from Cases A and B}

The method was tested in two cases, and it seems to identify a significant effect modification.

In Case A, the WE impact of the Intervention theme "new care concept," was assessed to be major. However, the WE impact of the two other Intervention themes were assessed as negative, suggesting no major changes in the WE due to the combined effect of the interventions. In contrast, the combined WE impact of the Modifier themes seems to have contributed to a pronounced impairment of the WE. Without effect modifier assessment the pooled impact of the investigated interventions may be interpreted to cause an impaired WE, although the true result presumably would be no impact.

In Case B, all the Intervention themes resulted in a major positive impact on the WE. However, the Modifier themes also seemed to have a major positive effect on WE, thus magnifying the true impact of the Intervention themes. Without using the EMA method, the pooled impact of the investigated interventions in this case would have been classified as true positive, but quantitatively exaggerated.

\subsection{Methodological considerations}

\subsubsection{Participants}

The EMA method involves employees in assessment of potential modifiers. Thus, it uses a participatory process. But, in contrast to "participatory ergonomics" (cf. Wilson, 1995), it is not the aim to involve the participants in designing, planning, or introducing the intervention(s). In fact, the participants in the EMA process should not have been involved in the previous intervention process as these individuals might be biased regarding the expected impact of the interventions. Further, managers should not participate as this may constrain the responses from the staff (Kitzinger, 1995). In the presented cases the EMA method was used following intervention processes based on participatory ergonomics engaging other employees. 
The EMA participants should be representative of all the occupational groups affected by the investigated intervention. We recommend two to five participants rather than eight to 14 , as suggested by Millward (2012). Each participant generally produces 8-12 post-it notes. More than five participants may result in a workshop that is too long in duration, not allowing each participant to exhaust his/her event assessments. However, a low number of participants may increase the risk of not identifying all significant events and/or cause the insufficient evaluation of these. To counteract this, participants should have broad experience at the ward, representing the largest possible part of the work performed in the investigated organization.

We anticipate that such bias will show as events that the participant expected but may not have happened or had to little impact to be perceived as an event by normalemployees.

At large wards, several workshops may be needed to obtain sufficient knowledge and evaluation of the significant events. However, this would obviously increase the amount of time required and, subsequently, the costs for both the investigated ward and the researchers.

\subsubsection{Definition of an Event}

An Event is defined as a change in the workplace/work perceived by the employees as having an impact on their work or work situation, thus being a potential risk factor. All events assessed as WE+ or WE- may potentially have an impact on musculoskeletal and/or mental health. Some of the events concern traditional ergonomic risk factors, e.g., heavy lifting (in case B, part of "new equipment and training"). Other events concern changes at the organizational system level, e.g., downsizing (in case A, "cutbacks"). Moreover, rumors may constitute risk factors if they create uncertainty, e.g., during organizational change (Bordia et al., 2004; 2006; Probst, 2003). A comprehensive study on risk factors at the organizational system level is presented and discussed by Westgaard and Winkel (2011).

\subsubsection{Assessment of Events and Themes}

The EMA method relies on three questions, which serve as a funnel from a very broad description of "important events" to the focused question regarding the impact that an event may have on the WE. The broad question aims at capturing all perceived significant events, and it does not narrow the participants' focus to just the WE. Thereby, the method may also detect events that are not immediately perceived by the participants as causes of changes in the WE.

The events that are mentioned during the first question may be considered most important, as they are not triggered by qualifiers such as "work environment" or "work processes and equipment." Further research is needed to investigate whether the responses to the three questions used in this study should be weighted differently.

Participants assess whether events are part of the intervention, and this is later reassessed by the researchers. This triangulation is important, as the participants may have insufficient knowledge about all interventions.

To obtain an overall assessment of events' impact, these are weighted in several steps. The mere occurrence of many events pointing to the same main event does not necessarily imply a high WE impact. Such events are therefore pooled into the same theme. The potential impact of a theme is 
then weighted according to amount of staff impacted, the duration of the impact, and the level of impact for these people during the period in question. Finally, the impact of the Modifier themes is compared with the impact of the Intervention themes. This comparison is assumed to be important, as studies of large ergonomic changes may be less sensitive to effect modifiers than those of minor ergonomic changes.

\subsubsection{Procedures to increase the validity of event assessment}

In intervention studies, the impact is commonly assessed by self-reports (e.g., questionnaires and diaries) from employees; this seems to be the most appropriate and cost-effective way to study a large number of individuals (Winkel and Mathiassen, 1994). The EMA method also uses self-reports, in part by proxy respondents. The sensitivity and specificity obtained by this procedure may be questioned. Due to this, we added dialogue between coach and participants and triangulations in the estimation of event and theme impacts on the WE. The validity of the assessments using this procedure needs to be investigated.

\subsubsection{The EMA method compared to alternative procedures}

We have not been able to identify other methods specifically aimed at modifier assessment. However, there are other methods that might be adapted to meet the specific needs for this purpose. Thus, "the process evaluation method" developed by Nielsen et al. (2006) and "the critical incident technique" developed by Flanagan (1954) might be used. The latter is a widely used qualitative research method applied across a diverse number of disciplines (for review, see Butterfield et al., 2005). However, both of these alternative methods seem to be considerably more time consuming in terms of field observations and interviews. The EMA method, as used in the two presented case studies, required less than two hours for the workshops. The time needed for the processing and analyses of collected data seems to be modest for the EMA method compared to the two other methods. Further, the EMA method aims at distinguishing between events part of and not part of the intervention. The two alternative methods integrate all events in their analysis.

\subsection{Limitations and opportunities}

The RCT study design is generally assumed to be the gold standard in intervention research, including ergonomics interventions (e.g., Driessen et al., 2010; Kennedy et al., 2010; Leyshon et al., 2010). Epidemiological quality is important in judging study quality. However, important insights are lost if inclusion in literature reviews of intervention research is based solely on such criteria. Studies of high epidemiological quality often lack detailed information on contextual factors that may affect the outcome and thus serve as modifiers, as also emphasized by Westgaard and Winkel (2011) and Westgaard (2010). On this background the present EMA method was developed.

In RCT studies, the control group may also be exposed to modifiers causing changes in the investigated outcome of this group. This may impede proper inferences regarding the impact of the investigated interventions. Therefore, control groups should also be investigated for potential effect modifiers.

The EMA method has so far only been tested in healthcare. The capacity of the employees to make valid assessments of significant events may vary between organizations. We presume that all employees are capable of assessing significant events at their workplace. However, the validity of this capacity may vary according to e.g. size of the organization and the organizational culture. Further validation of the method is therefore needed. 
The EMA method does not aim at considering potential effect modification due to differences in the way the intervention is delivered, if it is implemented, and the degree of compliance (Driessena, 2010; Nielsen et al., 2006; Rasmusen et al., 2017; Westgaard, 2010; Westgaard and Winkel, 1997; Winkel et al., 2015). It remains to be investigated whether the present EMA method can be further developed to estimate some of these issues as well.

The EMA method offers a systematic procedure for identifying and analyzing the effect on the studied outcome due to the intervention as well as modifier events. This may facilitate inference regarding the true impact of the investigated interventions. The EMA method should be thought of as a flexible set of principles that can be modified and adapted to meet the specific situation at hand (workplace context and investigated outcome). We believe the basic procedure could also be applicable in other types of research, e.g., in some types of evidence-based medicine, to add important contextual information and guard against effect modification (Victora et al., 2004; Greenhalgh et al., 2014).

\section{Conclusions}

- The present EMA method aims to assess effect modifiers in ergonomic intervention research. It focuses on changes in and around the investigated production system that may result in effects on musculoskeletal and mental health and/or the corresponding risk factors.

- The EMA method identifies both Intervention and Modifier events, i.e., potential risk factors, thus allowing a weighting of the modifier impact in relation to the intervention impact regarding the investigated outcome.

- The EMA method was tested in two cases illustrating how the modifiers' impact on the outcome may affect the conclusions regarding the impact of the interventions.

- The EMA method requires modest resources for data collection and analyses.

- The EMA method should be thought of as a flexible set of principles that can be modified and adapted to meet the needs in other types of evidence-based research to add important contextual information. This potential needs to be further investigated.

\section{Further Research}

The EMA method needs further evaluation; several issues to be investigated include:

- The three initial questions asked during an EMA workshop serve to funnel the focus of the study, i.e., changes in their WE. Should the responses to each of these questions be weighted differently?

- Only a few members of an organization participate in an EMA workshop. To what degree does this procedure mirror the assessments of all employees in the investigated organization?

- Two researchers rather than one are included in the triangulation procedures. Is the presumed gain in validity justified in relation to the increase in cost (researcher time)? 
- Does the EMA method offer reasonable validity? A relative estimate may be obtained by comparing the outcome from using the EMA method with more resource-demanding procedures, including interviews and field observations (i.e., inter-method reliability tests).

\section{Acknowledgements}

This study received financial support from the Danish Working Environment Research Fund (\#462010-09) and the healthcare authority in the county of VästraGötaland, Skaraborg Hospital, Sweden (\#SkaS 00203-2011).

The authors thank the investigated wards at Odense University Hospital for their good cooperation. A critical review of the manuscript has been completed by colleagues at DTU Management Engineering as well as Professor Emeritus Rolf Westgaard at the Norwegian University of Science and Technology, Trondheim, Norway.

\section{Declaration of interests}

None declared.

\section{References}

Bordia, P., Hobman, E., Jones, E., Gallois, C., Callan, V.J., 2004. Uncertainty during organizational change: types, consequences, and management strategies. Journal of Business and Psychologyaa, 18, 507-532.

Bordia, P., Jones, E., Gallois, C., Callan, V.J., Difonzo, N., 2006. Management are aliens! Rumors and stress during organizational change. Group Organization Management, 31, 601-621.

Driessen, M. T., Proper, K. I., van Tulder, M. W., Anema, J. R., Bongers, P. M., \& van der Beek, A. J. (2010). The effectiveness of physical and organisational ergonomic interventions on low back pain and neck pain: A systematic review. Occupational and Environmental Medicine, 67(4), 277-285. https://doi.org/10.1136/oem.2009.047548

Driessen, M. T., Anema, J. R., Proper, K. I., \& van der Beek, A. J. (2010). Authors' response: RCTs of ergonomic interventions. Occupational and Environmental Medicine, 67(4), 218-219.

https://doi.org/10.1136/oem.2009.050047

Parent-Thirion, A., Biletta, I, Cabrita, J., Vargas, O., Vermeylen, G., Wilczynska, A. \& Wilkens, M.. (2017). Eurofound (2017), Sixth European Working Conditions Survey - Overview report (2017 update), Publications Office of the European Union, Luxembourg

Flanagan, J. C., (1954). The critical incident technique. Psychological Bulletin, 51(4),

Greenhalgh, T., Howick, J., Maskrey, N., \& for the Evidence-Based Medicine Renaissance Group. (2014). Evidence-based medicine: A movement in crisis? BMJ, 348(jun13 4), g3725-g3725. https://doi.org/10.1136/bmj.g3725

Goldman, A. E. (1962). The group depth interview. The Journal of Marketing, 61-68. 
Jick, T. D. (1979). Mixing qualitative and quantitative methods: Triangulation in action. Administrative Science Quarterly, 24(4), 602-611.

Kennedy, C. A., Amick III, B. C., Dennerlein, J. T., Brewer, S., Catli, S., Williams, R., ... Rempel, D. (2010). Systematic review of the role of occupational health and safety interventions in the prevention of upper extremity musculoskeletal symptoms, signs, disorders, injuries, claims and lost time. Journal of Occupational Rehabilitation, 20(2), 127-162. https://doi.org/10.1007/s10926-009$\underline{9211-2}$

Keyte, B. \& Locher, D. (2004) The complete lean enterprise. Value stream mapping for administrative and office processes. Productivity Press, New York

Kitzinger, J. (1995). Qualitative research: Introducing focus groups. BMJ: British Medical Journal, 311(7000), 299.

Kristensen, T. S. (2005). Intervention studies in occupational epidemiology. Occupational and Environmental Medicine, 62(3), 205-210. https://doi.org/10.1136/oem.2004.016097

Leyshon, R., Chalova, K., Gerson, L., Savtchenko, A., Zakrzewski, R., Howie, A., \& Shaw, L. (2010). Ergonomic interventions for office workers with musculoskeletal disorders: A systematic review. Work, (3), 335-348. https://doi.org/10.3233/WOR-2010-0994

Limborg, H. J. \& Hvenegaard, H. (2011). The chronicle workshop: The use of narratives to reveal the story of a work community and understand its culture. In L. B. Rasmussen (Ed.) Facilitating change: Using interactive methods in organizations, communities and networks (pp. 305-325). Lyngby: Polyteknisk Forlag.

McLafferty, I. (2004). Focus group interviews as a data collecting strategy. Journal of Advanced Nursing, 48(2), 187-194.

Millward, L. J. (2012). Focus groups. In G. M. Breakwell, J. A. Smith, \& D. B. Wright (Eds.), Research methods in psychology (4th ed.) (pp. 411-438). Los Angeles: SAGE.

Nielsen, K., Fredslund, H., Christensen, K. B., \& Albertsen, K. (2006). Success or failure? Interpreting and understanding the impact of interventions in four similar worksites. Work \& Stress, 20(3), 272287. https://doi.org/10.1080/02678370601022688

Probst, T.M. (2003). Exploring employee outcomes of organizational restructuring - a Solomon fourgroup study. Group \& Organization Management, 28, 416-439

Rasmussen, C. D. N., Lindberg, N. K., Ravn, M. H., Jørgensen, M. B., Søgaard, K., \& Holtermann, A. (2017). Processes, barriers and facilitators to implementation of a participatory ergonomics program among eldercare workers. Applied Ergonomics, 58, 491-499.

https://doi.org/10.1016/j.apergo.2016.08.009

Rother, M. \& Shook, J. (2009). Learning to see: Value stream mapping to add value and eliminate MUDA (Version 1.4). Cambridge, MA: Lean Enterprise Institute.

Slavin, R.E. (1995). Best evidence synthesis: An intelligent alternative to meta-analysis. Journal of Clinical Epidemiology, 48(1), 9-18. https://doi.org/http://dx.doi.org/10.1016/0895-4356(94)00097-A 
Victora, C. G., Habicht, J.-P., \& Bryce, J. (2004). Evidence-based public health: Moving beyond randomized trials. American Journal of Public Health, 94(3), 400-405.

Westgaard, R. H. (2010). RCTs of ergonomic interventions. Occupational and Environmental Medicine, 67(4), 217-218.

Westgaard, R. H. \& Winkel, J. (1997). Ergonomic intervention research for improved musculoskeletal health: A critical review. International Journal of Industrial Ergonomics, 20(6), 463-500.

Westgaard, R. \& Winkel, J. (2011). Occupational musculoskeletal and mental health: Significance of rationalization and opportunities to create sustainable production systems-A systematic review. Applied Ergonomics, 42(2), 261-296.

Wilson, J. R. (1995). Solution ownership in participative work redesign - The case of a crane control room. International Journal of Industrial Ergonomics, 15(5), 329-344. https://doi.org/10.1016/01698141(94)00080-M, 10.1016/0169-8141(94)00080-M

Winkel, J., Edwards, K., Birgisdóttir, B. D., \& Gunnarsdóttir, S. (2015). Facilitating and inhibiting factors in change processes based on the lean tool "value stream mapping": An exploratory case study at hospital wards. International Journal of Human Factors and Ergonomics, 3(3/4), 291-302.

Winkel, J., Edwards, K., Birgisdóttir, B. D., Jarebrant, C., Johansson Hanse, J., Gunnarsdóttir, S., ... Ulin, K. (2015). A Nordic evaluation of a work environment complement to Value Stream Mapping for increased sustainability of patient flows at hospitals: The NOVO Multicentre Study I. Abstract Book 9th Novo Symposium, Quality in Health Care, 33-35.

Winkel, J. \& Mathiassen, S. E. (1994). Assessment of physical work load in epidemiologic studies: concepts, issues and operational considerations. Applied Ergonomics, 37(6), 979-988.

Winkel, J. \& Westgaard, R. H. (1996). Editorial: A model for solving work related musculoskeletal problems in a profitable way. Applied Ergonomics, 27(2), 71-77. 